\title{
Odorant quality perception: A metric individual differences approach
}

\author{
DANIEL B. KURTZ, PAUL R. SHEEHE, PAUL F. KENT, \\ THERESA L. WHITE, DAVID E. HORNUNG, and HERBERT N. WRIGHT \\ State University of New York Upstate Medical University, Syracuse, New York
}

\begin{abstract}
Perceptual spaces, in which similar stimuli are located close to each other and dissimilar stimuli are located far apart, have aided in the understanding of the physiological and psychological bases for sensory quality coding. Differences in perception between individuals should be reflected by differences in the spatial relationships between stimuli. If the dimensionality of the perceptual space is small (e.g., color space), individual differences that reflect specific pathologies are readily apparent from visual inspection. On the other hand, if the dimensionality of the perceptual space is large (as is proposed for odor space), visual inspection alone may not reveal individual differences in quality perception. The present work presents an information-theory-based method for quantifying individual differences in quality perception from perceptual confusion matrices. The ability of this method to quantify individual differences in quality perception is shown in a hypothetical example of specific anosmia. Finally, the method is applied to the examination of intrasubject consistency of odorant quality perception.
\end{abstract}

People differ in their perceptual representations of the world owing to a combination of genetic variation, pathology, and experience. One way to graphically express perceptual representations of the world is with perceptual spaces. In a perceptual space, stimuli that are similar in perceptual quality are located close together, and those that are dissimilar in perceptual quality are located far apart. Perhaps the best-known perceptual space is the one for color vision: the color wheel representing hues matched for brightness. In this space, similar hues are located closer to each other, and dissimilar hues are located proportionally farther apart. (The color chooser charts commonly found in personal computers present different forms of color space. However, the hues are not matched for brightness, nor are different hues positioned to appropriately represent the degree of perceptual dissimilarity.) Distortions in the color-wheel-like pattern of hues can occur in people with genetic conditions, such as color blindness, that arise from either one or more missing visual cones or the alteration of one or more of the photopigments. These conditions result in the well-known color confusions that typify each of the forms of this disorder. Identification and exploration of these confusions has aided in the understanding of visual color processing.

This experiment was presented in part at the 1994 International Symposium of Olfaction and Taste. The abstract of that presentation has been published in Chemical Senses, 1994, 19(5). Preparation of this article was supported by NIH Grant 9-PO1 DC00220. The authors thank Thomas E. Kurtz for helpful comments in the preparation of this manuscript and three anonymous reviewers and Associate Editor Jeremy Wolfe in the peer review process. Correspondence concerning this article should be addressed to D. B. Kurtz, SUNY Upstate Medical University, 750 East Adams Street, Syracuse, NY 13210 (e-mail: kurtzd@) mail.upstate.edu).
Color blindness results from the disruption of one or more of only three visual pigments (see Hurvich, 1981). As a consequence, color-blind individuals fall into but a few distinct groups: those lacking one, two, or three types of cones. If 10 or 20 different types of cones encoded color vision, deletion of one or more cone types would result in color blindness with many different clinical presentations. In this hypothetical situation, color blindness might be better described as a continuum, with people failing to fall into reasonably discrete groups. Such a complicated system would make difficult the task of inferring function from the naturally occurring pathologies of the system. Unfortunately, this hypothetical situation is manifest in the case of odorant perception, in which a family of 1,000 putative receptor molecules has been identified (Buck \& Axel, 1991) and in which many specific anosmias have been identified (Amoore, 1977).

Perhaps because odorant receptor types are so numerous, the structure of odorant quality space has remained elusive. However, some features of odorant quality perception appear across a number of studies. These features include perceptual intensity, hedonics (Schiffman, 1974), and irritation (Schiffman, Robinson, \& Erickson, 1977). Although these attributes have often been associated with olfactory stimuli, they arguably represent features that confound odorant quality perception. Perceptual intensity may be controlled by carefully choosing iso-intensive sets of odorants. Hedonic tone is idiosyncratic (Schiffman et al., 1977), whereas irritation largely reflects coactivation of the trigeminal nerve (Cometto-Muniz \& Cain, 1990). In a similar fashion, perceptual brightness and saturation are often fixed in investigations of color perception, to eliminate the effect of these variables on the dimensionality of color space. Even with some attributes held constant, the dimensionality of odorant quality space 
is unlike color space, in that it is dependent on the set of odorants chosen for investigation. It is possible that, as in color vision, odorant perceptual space has a dimensionality that is one less than the number of receptor types (three types of cones lead to a two-dimensional color space, the color wheel). If it were the case that odorant space has a large dimensionality ( 999 or so), the structure of odorant quality space might not be fully revealed until thousands of odorants have been investigated.

Although the dimensionality of odorant quality space remains unknown, in a conceptual odorant quality space, the distance between any two odorants represents the degree of perceptual similarity between those two odorants. Odorants that are closer together in space would be more perceptually similar. As perceptual similarity increases, confusions between the odorants is more likely to occur. The degree of confusion between odorants, therefore, indicates the degree of similarity between those odorants. This idea formed the basis for the odorant confusion matrix, or OCM (Kurtz, Hornung, Emko, \& White, 1996; Kurtz et al., 1995; Kurtz et al., 1994; Kurtz, White, \& Hayes, 2000; Wright, 1987), used in the clinical evaluation of dysosmic patients at the Smell and Taste Disorders Clinic (State University of New York Health Science Center at Syracuse). In these patients, incorrect odorant identifications (also known as confusions) represent a distortion of sensory perception. A central research question focuses on what relationships there are between olfactory pathology and distortions in odorant perception.

One alternative to the generation of an odorant quality space when examining issues of odorant quality perception is the placement of people in a space that reflects their responses to a given set of odorants. This people space can be thought of as reflective of a perceptual space, in that people who share a common perceptual view (similar perceptual spaces) tend to be grouped together and those who express different perceptual representations tend to be placed farther apart. That is, other things being equal, it is assumed that consistent alterations in odorant perception, whether these are due to nature, nurture, or experimental manipulation, would result in consistent displacement of people in people space.

O'Connell and his co-workers (O'Connell, Stevens, Akers, Coppola, \& Grant, 1989) chose a people space approach in their work on perceptual odorant channels. This was achieved by applying factor analysis to semantic descriptors produced by subjects with varying degrees of hyposmia to androstenone. Factor scores formed the basis of a three-dimensional people space, so that people who utilized similar descriptors for an odorant were grouped together and those who used different descriptors were farther apart. This approach allowed the authors to conclude that a series of perceptually similar odorants were encoded by a number of separate perceptual channels. Schiffman has also examined individual differences in odorant quality perception. In this work, odorant object space was created by applying "individual" multidimensional scaling (MDS) to ratings of odorant dis- similarity. Although this MDS routine produced a single odorant space, the analysis also determined how strongly each subject utilized each of the dimensions of the joint odorant object space. That is, differences between individuals in odorant perception were manifested in the strength each subject placed on each of the dimensions in the multidimensional odorant object space. In numerous studies, Schiffman has shown that these weightings are consistent across normal subjects (Schiffman \& Dackis, 1976) and vary in a consistent fashion with aging (Schiffman \& Leffingwell, 1981) and with obesity (Schiffman, Musante, \& Conger, 1978). Here again, rather than direct evaluation of odorant object space itself, groups of people were used to infer differences in odorant perception.

The lines of investigation described above allowed for the visual representation of differences between groups of people. By inference, these differences reflected underlying differences in odorant quality perception. However, the people spaces in the literature did not possess metric properties. That is, differences between people were not directly interpretable, because distances along one dimension may have carried different meanings than distances along other dimensions. The purpose of the present work is to develop a methodology that will generate spaces in which euclidean distances can reflect the degree of difference in odorant quality perceptions of people or groups of people. (Note, the term euclidean distance is used to denote the usual straight line distance between points in euclidean space. This term is used to distinguish the present understanding of distance from other possible measures, such as city block distance.)

The purpose of this work is to extend the concept of perceptual dissimilarity spaces to the understanding of olfaction and, specifically, to the investigation of olfactory pathology, while avoiding the necessity of understanding either the structure or the dimensionality of the perceptual space. A people space approach could relate differences in OCM response patterns to the various causes of olfactory loss in different groups of people. To create a people space, the present work presents a method by which to quantify the overall dissimilarity between two OCMs. A low-dimensionality people space is generated by MDS, thereby allowing visual inspection of the placement of individuals in this space. Shifts in this space, therefore, reflect differences in odorant quality perception. In addition, the resulting multidimensional patterns are analyzable by the direct extension of conventional analysis of variance (ANOVA) procedures. The method is then demonstrated by the creation of a hypothetical specific anosmia and through an investigation of withinsubject consistency of odorant quality perception. The techniques discussed should be applicable to investigations of individual differences of perception in any sensory system.

\section{The Odorant Confusion Matrix}

The OCM characterizes responses to olfactory stimuli by examining the distribution of odorant-naming re- 
Table 1

OCM Stimuli and Odorant Dilutions

\begin{tabular}{|c|c|c|c|c|}
\hline Odorant & Solute & Source & Purity & Dilutions \\
\hline Ammonia & ammonium hydroxide & Aldrich & $29.1 \%\left(\mathrm{NH}_{3}\right)$ & $6.3 \% \mathrm{v} / \mathrm{v}$ \\
\hline Cinnamon & trans-cinnamaldehyde & Aldrich & $99 \%$ & $1.6 \% \mathrm{v} / \mathrm{v}$ \\
\hline Licorice & trans-anethole & Aldrich & $99 \%$ & $0.19 \% \mathrm{v} / \mathrm{v}$ \\
\hline Mint & R-carvone & Aldrich & $98 \%$ & $6.3 \% \mathrm{v} / \mathrm{v}$ \\
\hline Mothballs & naphthalene & Aldrich & $99 \%$ & $0.63 \% \mathrm{w} / \mathrm{v}$ \\
\hline Orange & $d$-limonene & Sigma & $97 \%$ & $0.19 \% \mathrm{v} / \mathrm{v}$ \\
\hline Rose & phenethyl alcohol & Sigma & $99.4 \%$ & $12.5 \% \mathrm{v} / \mathrm{v}$ \\
\hline Rubbing alcohol & 2-propanol & Fisher & $99.9 \%$ & $12.5 \% \mathrm{v} / \mathrm{v}$ \\
\hline Vanilla & vanillin & Aldrich & $99 \%$ & $0.42 \% \mathrm{w} / \mathrm{v}$ \\
\hline Vinegar & acetic acid & Aldrich & $99.8 \%$ & $25 \%$ \\
\hline Vex & (diluent: 1,2-propane diol) & Sigma & $99.9 \%$ & \\
\hline
\end{tabular}

sponses. As the procedure for administering the OCM has been described carefully elsewhere (Wright, 1987), it will be reviewed only briefly. The odorants and concentrations making up the OCM have changed over the years and, therefore, will be described in more detail. The odorant names, chemical names, and dilutions are presented in Table 1. The odorants are presented in $30-\mathrm{ml}$ amber glass bottles (Thomas Scientific, 9711E78) and diluted in 1,2-propanediol to a total volume of $15 \mathrm{ml}$.

To generate each confusion matrix, a subject attempts to identify each of 10 odorant stimuli (plus a blank, known as a vex) from a list of 10 odorant names. These 11 stimuli are given to the subject in 11 randomized blocks, for a total of 121 stimulus presentations. Unknown to the subject, each odorant is presented once in each block, with no odorant following itself in the presentation order. Also unknown to the subject is that the first block of 11 odorants is for practice only and the data collected are discarded. The subject is not told of the presence of the vex and is required to respond to all the stimuli with an odor name chosen from a list. This list of names is visible to the subject at all times. The subject is given the freedom to sniff the odorants in whatever manner seems best and as often as necessary. The subject's record of responses to the odorants is placed in a matrix (stimulus vs. responses), in which tallies (responses) on the main diagonal represent correct identifications and tallies on the off-diagonal represent misidentifications.

\section{An Informational Measure of Dissimilarity}

Two odorants might be considered to be similar in perceptual quality if the two odorants are confused with each other. In addition, two odorants that are confused with a common third odorant could also be considered similar in quality. The distribution of a subject's responses to a given odorant is influenced by that subject's perception of the odorant at the time of presentation. The distribution of a 2 nd subject's responses to the same odorant provides an indication of the similarity or dissimilarity between the perceptions of the 2 subjects. Accordingly, a metric based on average information transmitted (Attneave, 1959) was developed that quantified response consistency on the OCM across people.
The dissimilarity between two OCMs is measured by $D$. The form of each OCM is a $10 \times 10$ matrix of frequencies $\left(f_{i j k}\right)$ of response $j$ by subject $k$ to stimulus $i$, with $i=1,2, \ldots, 10, j=1,2, \ldots, 10$, and $k=1,2$ (as in the example below in which the consistency of odorant quality perception was examined, $k$ may also represent multiple tests performed within the same subject). With respect to a particular stimulus/odorant $(i)$, the dissimilarity between the distributions of responses by the 2 subjects is measured by the average information transmitted by their responses:

$$
D_{i}=\sum_{j k}\left(P_{i j k} \times \log _{2} P_{i j k}\right)-\sum_{j}\left(P_{i j .} \times \log _{2} P_{i j .}\right)+1,
$$

where $P_{i j k}=f_{i j k} / f_{i} \ldots$. The overall measure of dissimilarity is

$$
D=\sum_{i} D_{i} .
$$

That is, the dissimilarity between any two matrices is the sum (across the 10 odorants) of the dissimilarities between the patterns of responses for each particular odorant in one matrix and for the same odorant in the other matrix.

\section{Example of Hypothetical Specific Anosmia}

The purpose of the above measure is to quantify individual differences in the pattern of odorant identification and misidentification. To examine the face validity of this approach, the following demonstration was created. Thirty-five subjects were selected from a large database of patient OCM results collected over the past 10 years and served as test subjects. Normosmic $(80 \%$ correct or greater on the OCM) subjects were selected such that their binasal performance level (or percent correct) fell between $80 \%$ and $90 \%$. The subjects were arbitrarily subdivided into three groups, called Vinegar, Mothballs, and Rose (the purpose of which will be evident below). The dissimilarity between each subject's OCM and the OCM of each of the other subjects was determined, resulting in a $35 \times 35 \mathrm{ma}$ trix of pairwise dissimilarities. To reduce the dimensionality of this matrix, the data were subjected to MDS (SYSTAT, Kruskal, linear). The resulting two-dimensional 


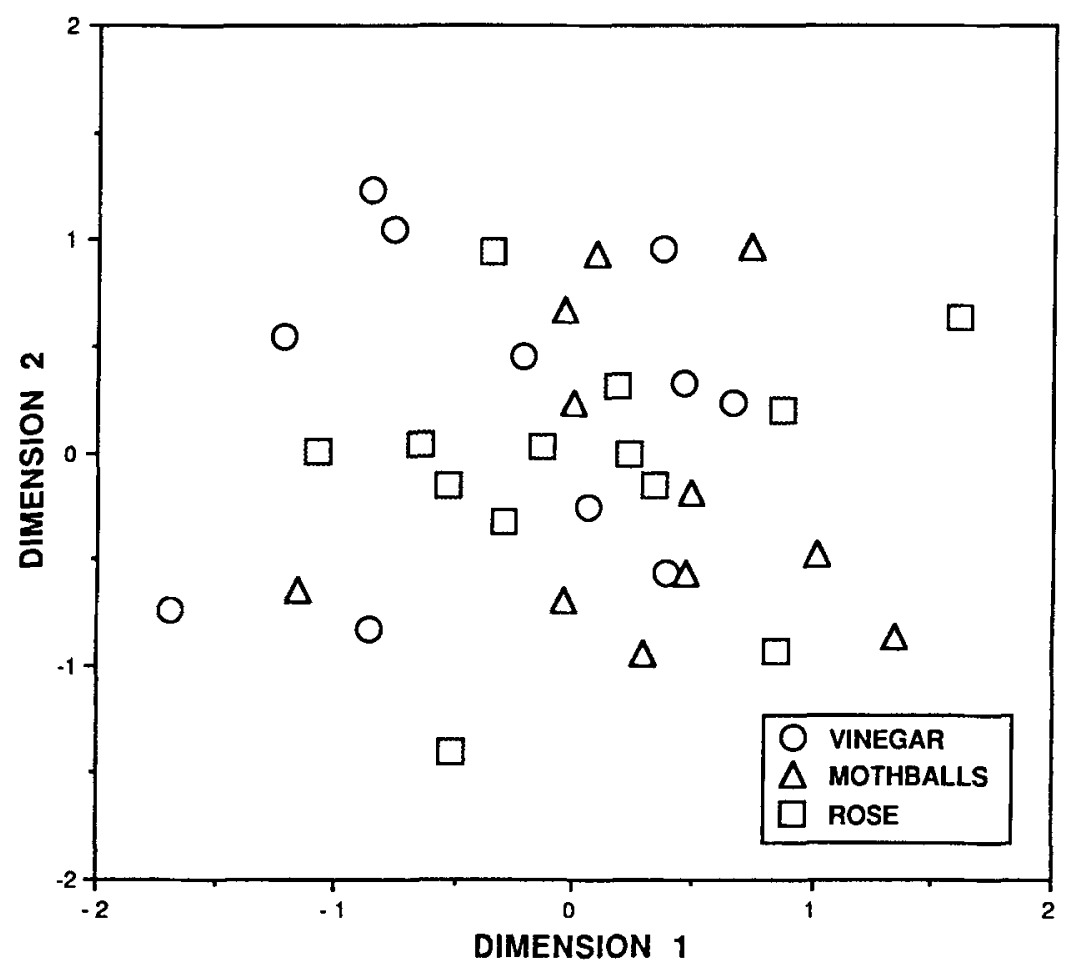

\begin{abstract}
Figure 1. Random-like distribution of normal subjects, labeled Vinegar, Mothballs, and $R o s e$, in two-dimensional people space. This space was created by (1) selecting the odorant confusion matrices (OCMs) from 35 normosmic patients who served as test subjects, (2) calculating the dissimilarity $(D)$ between $O C M$ from every pair of subjects, and (3) creating a two-dimensional people space by applying multidimensional scaling to the $35 \times 35$ triangular dissimilarity matrix. The position of people appears random.
\end{abstract}

people space (Figure 1) resembles a random-like organization of the three groups of normosmic subjects.

For each group of subjects, responses to a particular odorant were replaced with each subject's own responses to the vex. The responses to the vex were chosen in an effort to mimic a specific anosmia in which each subject was unable to perceive one particular odor. (Note: The extrapolation to an understanding of specific anosmia itself should be done carefully, in at all. Loss of sensitivity to a particular odorant likely involves many odorant receptor mechanisms. Loss of one or more receptor mechanisms would likely result in changes in odorant perception across a wide range of odorants.) For the first group, the vex responses were substituted for vinegar. The substitution was made for mothballs in the second group and for rose in the third group. Each subject's new matrix was then calculated, and the dissimilarity between matrices was calculated in the manner described above.

Although it could not be assumed a priori that intersubject variability in response pattern would allow a clear separation between the three groups of subjects, it is readily apparent from a visual comparison of Figures 1 and 2 that this approach allowed the $100 \%$ accurate separation of people on the basis of the type of specific anosmia. This separation was accomplished both in the presence of response error made in the original matrix and in the presence of variability in the responses to the vex.

\section{A People Space Approach to the Examination of the Short-Term Consistency of Odorant Object Space in Dysosmic Subjects}

Perhaps a more critical evaluation of this approach to individual differences in odorant quality perception is a comparison of intrasubject and intersubject variability. Common experience in sensory perception suggests that quality perception is relatively consistent within a person across time. That is, the color of an object usually does not appear to change from moment to moment (given that the stimulus is constant). It is likely that the same is true for odors, although, arguably, the consistency of odorant quality may be more difficult to recall from personal experience. However, basically, cinnamon smells like cinnamon each and every time the spice jar is opened. On the other hand, quality perception can be grossly different from subject to subject (e.g., color blindness and specific anosmia). The relative stability of odorant perception within people across time versus the relative variability across people, then, served as a way to evaluate the people 


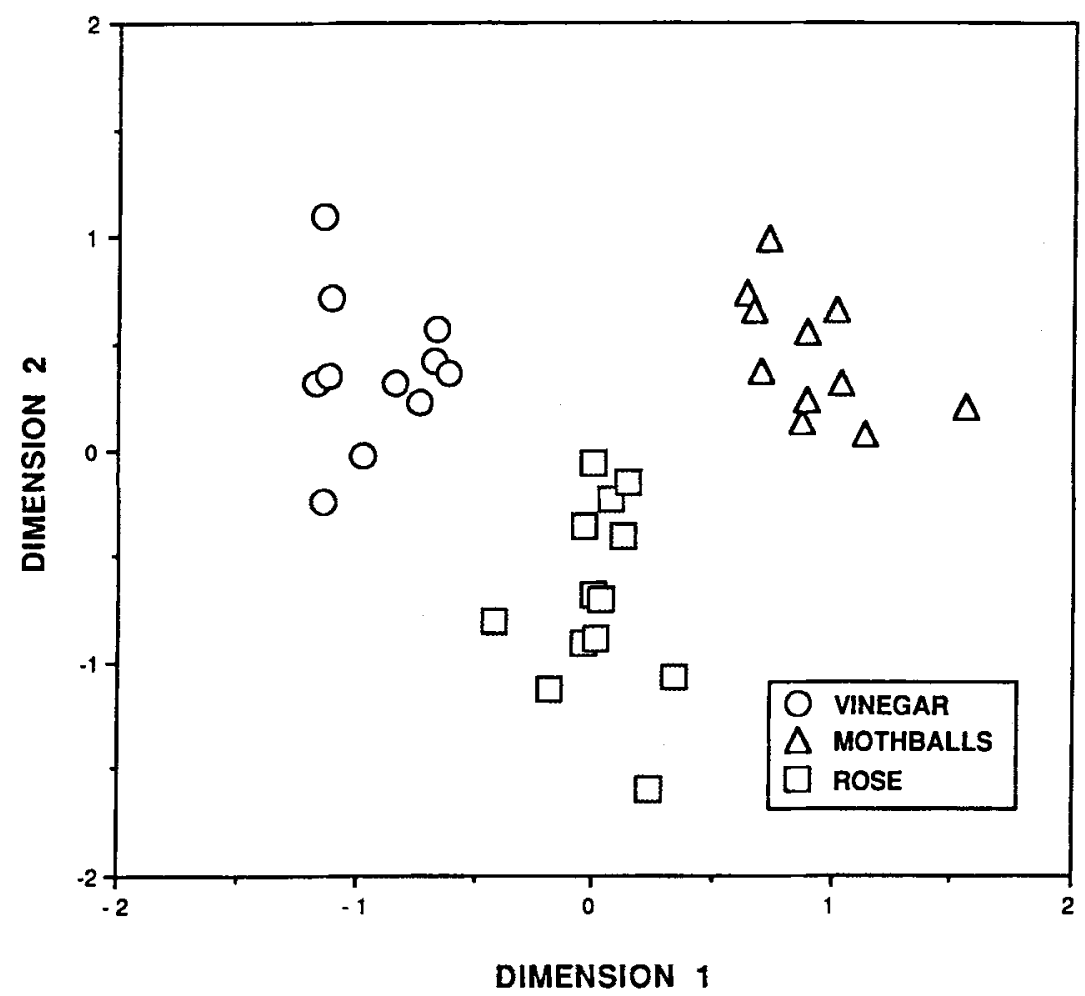

Figure 2. Segregation of subjects with the vex substituted for responses to vinegar, mothballs, and rose in two-dimensional people space. For the 35 subjects in Figure 1, the responses to the vex for each subject were substituted for the actual responses to either vinegar, mothballs, or rose to simulate a loss of each of the odorant qualities. As in Figure 1, the dissimilarity between each pair of the new matrices was calculated, and a two-dimensional people space created with multidimensional scaling. People have been segregated on the basis of the odorant to which they have become anosmic.

space approach as a technique with which to examine differences in their odorant object spaces.

\section{METHOD}

\section{Subjects}

Twenty patients ( 11 males and 9 females; mean age $=53.1, S D=$ 17.3), who presented to the Smell and Taste Disorders Clinic, agreed to be tested twice with the OCM as part of a clinical evaluation for dysosmia and, thereby, served as test subjects. The subjects were hyposmic, with OCM scores covering the entire range of this category $(20 \%-79 \%$ correct $)$. The etiologies of the olfactory losses in these subjects reflected most of the major causes of olfactory loss-- for example, head trauma, polyposis, upper respiratory infection, and so forth. All the subjects provided written informed consent under a protocol approved by the institutional review board (IRBPHS, SUNY Health Science Center at Syracuse).

\section{Procedure}

The procedure for administering the OCM was similar to that described above. Each subject was given the OCM binasally on two occasions (Test 1 and Test 2). The two tests were separated by about $2 \mathrm{~h}$, with a routine ENT examination (external manual exam, oral visual exam, rigid nasal endoscopy without touching the mucosal membranes of the nasal cavity) performed between the two tests (testing for 2 subjects was separated by 1 day).

\section{RESULTS}

Average percentages correct on the first and second OCMs were not significantly different (first test $=44.4 \%$, second test $=44.7 \%$; paired $t$ test, $t=0.14, p=.88$ ). In addition, percentages correct on the first and second tests were highly correlated $\left(r^{2}=.837, p<.001\right)$. These results reflect the relative stability of the olfactory performance level within subjects but do not address the stability of odorant perception itself. That is, when odorants are misnamed, do hyposmic subjects make errors (confusions) that are reliability consistent over time?

To address this question, the dissimilarity between the Test 1 and the Test 2 matrices produced by each subject was quantified by $D$, as was the dissimilarity between each matrix for a subject and each matrix of each of the other subjects. This resulted in a $40 \times 40$ matrix of pairwise dissimilarities. The distributions of these dissimilarities are presented separately for within-subject dissimilarity and for between-subjects dissimilarity in Figure 3. The absolute frequencies of within-patients dissimilarities represent but a small fraction of the between-subjects dissimilarities. The within-subject dissimilarity generally fell 


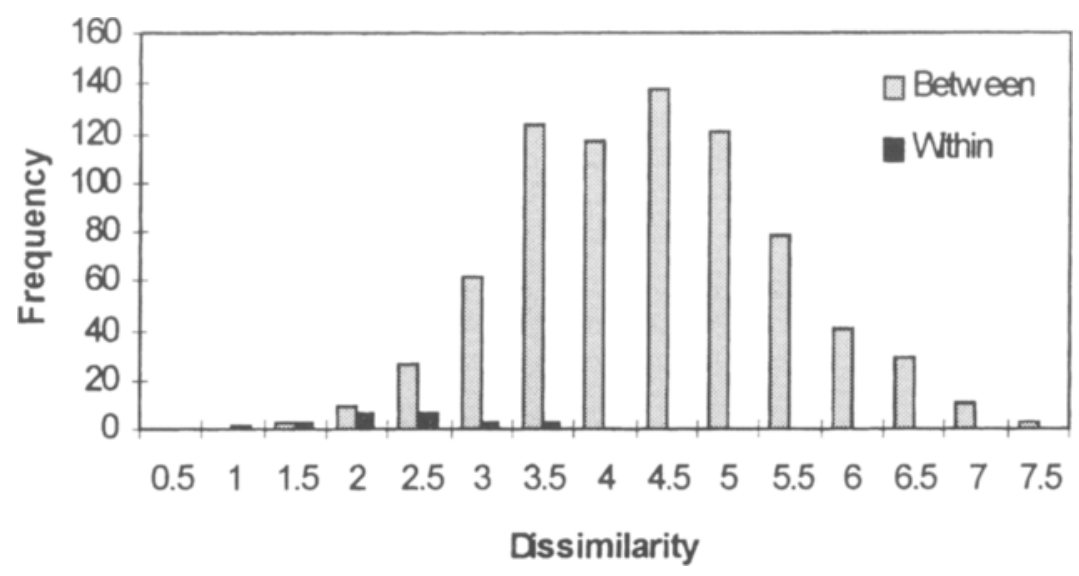

Figure 3. Distribution of dissimilarities. The dissimilarity $(D)$ between all pairs of the 40 odorant confusion matrices (OCMs) (2 OCMs from each of 20 hyposmic patients) was calculated. The dissimilarities between each subject's first and second OCM (representing intrasubject variability) were generally smaller than the dissimilarities between each subject and every other subject (representing intersubject variability).

below the first quartile of all between-subjects dissimilarities. The mean within-subject dissimilarity $(2.02)$ was less than half of the mean between-subjects dissimilarity (4.16). That is, within-subject variability was considerably smaller than between-subjects variability, a result that is consistent with the assumptions noted above.

$D$ can be thought of as representing two features of the confusion matrix - namely, correct odorant identification and incorrect odorant identification or odorant confusions. As was described above, incorrect odorant identification can be a reflection of odorant perceptual similarities. The consistency of odorant confusions within people is important in the determination of whether patients with different etiologies of olfactory loss can be discriminated on the basis of odorant quality perception. To separate the influence of percent correct from quality perception per se in the evaluation of inter- and intrasubject variability, the dissimilarity matrix was subjected to MDS (SYSTAT, Kruskal, linear; Kruskal stress $=0.108$ ).

To evaluate how well the MDS preserved the metric properties of $D$, the OCM dissimilarity measure, the relation between the input dissimilarity data and the output five-dimensional euclidean distance was evaluated. The Shepherd plot of this relationship (Figure 4) suggests a linear relationship. This impression is supported by a strong linear regression component $\left(R^{2}=.899\right)$ and a weak quadratic component (regression of the square of dissimilarity as the independent variable, with the residuals from the previous linear regression as the dependent variable: $R^{2}=$ $.0003)$. Therefore, distances in five-dimensional space are predominantly a linear function of calculated matrix dissimilarities, thereby allowing MDS euclidean distances in five-dimensional space to be interpreted directly as closely representing matrix dissimilarities with the metric properties of the dissimilarity measure.

In a fashion similar to that seen with the starting metric, $D$, euclidean distances between each subject's Test 1 and Test 2 were generally smaller than the euclidean distances across subjects - a result that reflects the previous finding and the preservation of the metric properties of $D$. Regressions performed on each of the five dimensions show that performance (percent correct) was grossly related to position in only the first dimension (dimension $1, R^{2}=.853$; dimension $2, R^{2}=.004$; dimension 3 , $R^{2}=.016$; dimension $4, R^{2}=.017$; dimension $5, R^{2}=$ $.001)$. In a general sense, this people space is structured so that the first dimension is predominately related to performance level on the OCM (percent correct) and the other four dimensions are not. By exclusion, the other four dimensions must be related to patterns of odorant identification and misidentification or, more globally, odorant quality perception. Because of this, the within-subject stability of odorant quality perception as separate from stability of performance level could be evaluated by comparing euclidean distances in dimensions $2-5$ between Tests 1 and 2. However, the segregation of performance level from odorant quality perception is only partially accomplished by the segregation of dimension 1 from dimensions 2-5. (The segregation of performance level or percent correct from quality perception is not as clear as is implied here. Correct odorant identification suggests that the odorant quality is consistent with the odorant quality retrieved from memory for that odorant name. Certainly, this implies an odorant percept that is relatively constrained. However, across odorants any given level of performance does not imply any one percept for all the odorants. Therefore, although higher levels of performance imply that each odorant is moving toward a particular percept, it does not necessarily describe that percept.)

To address the intrasubject stability of odorant quality perception more precisely, the effect of performance level was removed by including performance (log odds transformation of percent correct) as a covariate in a general linear model extension of an approach described by 


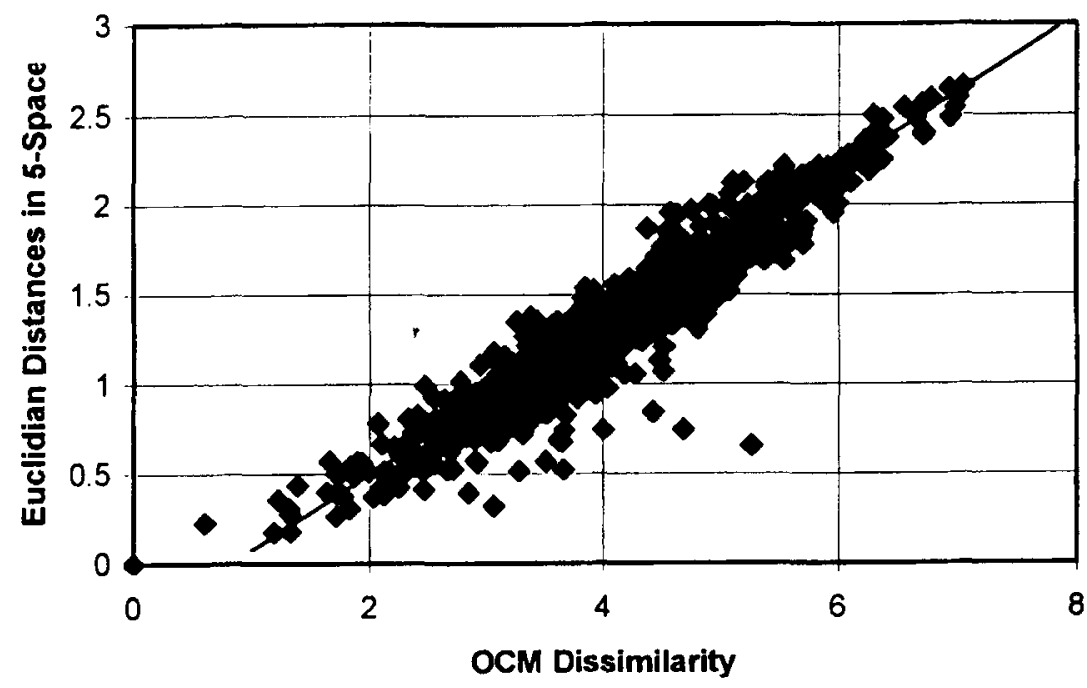

Figure 4. Euclidean distances from Kruskal multidimensional scaling (MDS) as a function of matrix dissimilarity. To evaluate the intrasubject consistency of odorant quality perception, the dimensionality of the dissimilarities generated for Figure 3 was reduced with MDS. To evaluate whether the metric properties of $D$ were preserved during data reduction, the input dissimilarities were plotted against the output distances in fivedimensional space in a Shepherd diagram. The linear nature of this relationship is evidence that the metric properties of $D$ have not been altered by fitting the 40 odorant confusion matrices (OCMs) from 20 people's Tests 1 and 2 into five-dimensional space.

Snedecor and Cochran (1989, and see the Appendix). In this approach, reliability $\left(\rho^{2}\right)$ is the ratio of the variance attributable to differences between subjects to the sum of that variance and the intrasubject variance. As a consequence, this measure can be thought of as a coefficient of determination and represents the relative stability of responses within subjects over time. The maximum likelihood estimate of reliability of patterns of odorant identification/misidentification was .8211 , with $95 \%$ confidence limits of .604 and .9246 . This is in the same neighborhood as the within-subject $R^{2}$ of percent correct reported above. That is, both overall performance and odorant quality perception as indicated by the pattern of responses show a high level of intrasubject stability that confers a high level of reliability in detecting intersubject differences.

\section{DISCUSSION}

The relative variability within and across subjects was preserved throughout the analysis of short-term consistency of odorant perceptual space. Percent correct odorant identification was stable within subjects over the $2 \mathrm{~h}$ between the two test sessions. This consistency was mirrored in the metric $D$, which quantified the dissimilarity between matrices, in that the average dissimilarity between Test 1 and Test 2 within a subject was considerably smaller than the dissimilarity between matrices of different subjects. After MDS and removal of percent correct, odorant quality perception was also more stable within a subject than across subjects. This demonstration of intrasubject stability in olfactory performance and odorant quality perception is consistent both with personal experience and with descriptions from subjects seen at the Smell and Taste Disorders clinic.

Odorant quality perception can be quantified with a number of different methodologies. The OCM developed by Wright (1987) is, at its primary level, an odorant identification task. Assigned odorant names can be thought of as correct or incorrect. In this embodiment, the OCM is similar to many other tests of olfactory ability-for example, the CCCRC (Cain, Gent, Goodspeed, \& Leonard, 1988), UPSIT (Doty, Shaman, \& Dann, 1984), and the Virginia Olfactory Screening Test (Costanzo \& Zasler, 1991). However, the OCM allows inferences to be made concerning odorant quality perception. Odorant naming is the process of matching an odor percept to that of an odor memory. Presumably, this process is based on similarity between the percept and the memory. The frequency with which a particular odorant name is assigned to a given odorant represents the degree of similarity between the percept and the memory. Indeed, to examine this implication in reverse, Getty, Swets, Swets, and Green (1979) accurately predicted a confusion matrix for complex sounds from similarity judgments. The application of confusion matrices to the understanding of perceptual space (odorant, sounds, etc.) is critically dependent on the presence of errors in the matrix. It is impossible to apply these techniques when performance is perfect, and difficult when performance is nearly perfect. For instance, in the OCM, if all the odorants were named correctly every time, each odorant would be mutually dissimilar. If a num- 
ber of subjects performed in a similar fashion, the intraand between-subjects variability would both be zero. In such cases, the stability of odorant perceptual space would more appropriately be investigated by other measures of perceptual dissimilarity (see, e.g., Kurtz et al., 2000, and Wise, Cain, \& De Wijk, 1997, for examples in olfaction).

The present work centers on a process for inferring differences in odorant perceptual space without actually generating that space. This analysis was performed by examining the consistency of odorant naming (and by inference, odorant object space) between and within people. The metric, $D$, quantified the dissimilarity of 1 subject's matrix from that of all the other matrices. Odorant object patterns that are similar are expected to produce confusion matrices that have similar distributions of odorant identification and misidentification. The stability of odorant naming within subjects was seen in the small dissimilarities between the matrices within subjects in relation to the dissimilarity of matrices across subjects.

The measure of dissimilarity between matrices, $D$, was developed with ratio scale properties. As was shown above, MDS euclidean distances were strongly linearly related to the input matrix dissimilarities. This allows MDS distances to be interpreted directly as matrix dissimilarities, with the metric properties of the dissimilarity measure reflected directly in euclidean distances in the MDS five-dimensional space.

Experience evaluating dysosmic patients in a clinical setting suggests that two basic types of olfactory loss occur. It appears that the pathology results in either shifts in odorant delivery (congestive olfactory loss, such as allergies, colds, nasal sinus disease, or polyposis) or neural damage. In some situations, an odorant may be named correctly on the OCM $30 \%-70 \%$ of the time, with the other responses distributed across other possible odorant names. That is, when the odorant is misnamed, it appears that the percept is not strong enough to allow the assignment of a particular quality and, therefore, name. This is a situation similar to that modeled earlier in the example. In other situations, an odorant is named consistently, but the name supplied is incorrect. That is, mint might be called licorice, or rose called mothballs. In these situations, it appears that the olfactory code has been altered (see Schiffman, 1992), the subject has a response bias, or both. In continuing work on the OCM, we hypothesize that some disease processes produce distinct alterations in the code representing particular odorants, resulting in the unique odorant confusions seen in the OCM. If the code for different odorants is varied, their relative positions in the odorant space should also be altered. This alteration should be reflected in changes in the pattern of odorant identification and misidentification in the OCM, and, thus, people should be positioned differentially in people space. To demonstrate the relationship between disease processes and olfactory dysfunction, changes in the position of people in people space will be examined as a function of the disease state. The question to be addressed would be whether patients with similar etiologies of ol- factory loss group together in different multidimensional people space locations (as in Figure 2). Indeed, preliminary reports suggest that this tool does identify a number of etiologies of hyposmia that result in consistent placement of patients in multidimensional people space; therefore, they suggest that different etiologies of olfactory loss produce distinct alterations of odorant quality (Kurtz et al., 1995).

\section{REFERENCES}

AMoOre, J. E. (1977). Specific anosmia and the concept of primary odors. Chemical Senses \& Flavor, 2, 267-281.

ATTNEAVE, F. (1959). Applications of information theory to psychology. New York: Holt.

BUCK, L., \& AXEL, R. (1991). A novel multigene family may encode odorant receptors: A molecular basis for odor recognition. Cell, 65 , 175-187.

Cain, W. S., Gent, J. F., Goodspeed, R. B., \& Leonard, G. (1988). Evaluation of olfactory dysfunction in the Connecticut Chemosensory Clinical Research Center. Laryngoscope, 98, 83-88.

Cometto-Muniz, J. E., \& Cain, W. S. (1990). Thresholds for odor and nasal pungency. Physiology \& Behavior, 48, 719-725.

Costanzo, R. M., \& ZasLer, N. D. (1991). Head trauma. In T. V. Getchell, R. L. Doty, L. M. Bartoshuk, \& J. B. Snow, Jr. (Eds.), Smell and taste in health and disease (pp. 711-730). New York: Raven.

Doty, R. L., Shaman, P., \& DanN, M. (1984). Development of the University of Pennsylvania Smell Identification Test: A standardized microencapsulated test of olfactory function. Physiology \& Behavior, 32, 489-502.

GetTy, D. J., Swets, J. A., Swets, J. B., \& Green, D. M. (1979). On the prediction of confusion matrices from similarity judgments. Perception \& Psychophysics, 26, 1-19.

Hurvich, L. M. (1981). Color vision. Sunderland, MA: Sinauer.

Kurtz, D. B., Hornung, D. E., Emko, P. E., \& White, T. L. (1996). Relative odorant identification in the evaluation of hyposmia [Abstract]. Chemical Senses, 21, 628.

Kurtz, D. B., Hornung, D. E., Kent, P., Emko, P. E., White, T. L., \& SHEEHE, P. R. (1995). Odorant dissimilarity as a measure of changes in the human olfactory system: Implications for the evaluation of dysosmia. Chemical Senses, 20, 726-727.

Kurtz, D. B., Sheehe, P. R., Kent, P. F., Hornung, D. E., White, T. L., Youngentob, S. L., SChwo8, J. E., Mozell, M. M., Belknap, E. M., \& Eмко, P. E. (1994). Odorant confusion matrix response patterns: Relations to medical history [Abstract]. Chemical Senses, 19, 501.

Kurtz, D. B., White, T. L., \& HAYEs, M. (2000). The labeled dissimilarity scale: A metric of perceptual dissimilarity. Perception \& Psychophysics, 62, 152-161.

O'Connell, R. J., Stevens, D. A., Akers, R. P., Coppola, D. M., \& Grant, A. J. (1989). Individual differences in the quantitative and qualitative responses of human subjects to various odors. Chemical Senses, 14, 293-303.

SCHIFFMAN, S. S. (1974). Contributions to the physiochemical dimensions of odor: A psychophysical approach. In W. S. Cain (Ed.), Odors, evaluation, utilization, and control (Annals of the New York Academy of Sciences, Vol. 237, 164-183). New York: New York Academy of Sciences.

SCHIFFMAN, S. S. (1992). Olfaction in aging and medical disorders. In M. Serby \& K. L. Chobor (Eds.), Science of olfaction (pp. 500-525). New York: Springer-Verlag.

SCHIFFMAN, S. S., \& DACKIS, C. (1976). Multidimensional scaling of musks. Physiology \& Behavior, 17, 823-829.

Schiffman, S. S., \& Leffingwell, J. C. (1981). Perception of odors of simple pyrazines by young and elderly subjects: A multidimensional analysis. Pharmacology, Biochemistry \& Behavior, 14, 787 798.

Schiffman, S. S., Musante, G., \& Conger, J. (1978). Application of multidimensional scaling to ratings of foods for obese and normal weight individuals. Physiology \& Behavior, 21, 417-422. 
SChiffman, S. S., Robinson, D. E., \& Erickson, R. P. (1977). Multidimensional scaling of odorants: Examination of psychological and psychochemical dimensions. Chemical Senses \& Flavor, 2, 375-390.

Snedecor, G. W., \& Cochran, W. G. (1989). Statistical methods (8th ed.). Ames: Iowa State University Press.

WISE, P. M., CAIN, W. S., \& DE WIJK, R. (1997) Latency to discriminate as a measure of similarity of odor quality [Abstract]. Chemical Senses, 22, 823 .

WRIGHT, H. N. (1987). Characterization of olfactory dysfunction. Archives of Otolaryngology, 113, 163-168.

\section{APPENDIX \\ Within-Subject Reliability of OCM Response Patterns as Represented in MDS Test/People Space}

As was described in the main text, dissimilarity measures $(D)$ between each OCM of 20 hyposmic subjects produced $20 \times$ $2=40$ points in a five-dimensional MDS euclidean space that is interpreted to have an identity distance metric. In a general linear model that employs predictive measures and/or indicator variables, a series of conventional multiple regressions using each spatial coordinate as the dependent variable produces estimates of the dependent five-dimensional vectors. For each point in multidimensional space, the sum of squared errors in estimating the coordinate values produces the squared distance between the observed and the estimated points. With the orientation of the error vector ignored, this squared distance is interpretable as a univariate squared error. Consequently, the coordinate mean squares for the regression components and errors combine to produce multidimensional variance estimates that are tested by conventional ANOVA and analysis of covariance (ANCOVA) procedures. We apply these procedures to the $20 \times 2$ design of this study in order to estimate the reliability of OCM response patterns after removing the effects of different levels of percent correct.

Snedecor and Cochran (1989) define the reliability of a onedimensional response as the proportion of subject-to-subject variance that is not attributable simply to random-like withinsubject variability. That is, reliability is interpretable as a coefficient of determination-the squared correlation coefficient between observed responses and the error-free ("true") levels of responses of the subjects.

In our study, we extend the definition of reliability to apply to representations of OCM patterns of response in multidimensional space. Moreover, because the focus of our study is on the reliability of OCM response patterns after removing the effects of differences in levels of performance, we apply a covariate adjustment based on the log odds transform of percent correct.

Finally, we develop a maximum likelihood estimate $($ mle $)$ of reliability together with $95 \%$ confidence limits. In characterizing the ability of the OCM to discriminate among subjects, it is not sufficient to merely test a null hypothesis. Instead, confidence limits provide the necessary degree of precision in estimating the reliability of the OCM patterns.

\section{The Model}

Let $Y_{i j k}=\mu_{k}+\delta_{j k}+\beta_{k} x_{i j}+\varepsilon_{i j k}$, where $Y_{i j k}=$ the MDS coordinate value in dimension $k=1,2, \ldots, 5$ for subject $j=1,2, \ldots$, 20 in test $i=1,2 ; \mu_{k}=$ the expected value of points in dimension $k ; \delta_{j k}=$ the expected value of the deviation of subject $j$ 's response from $\mu_{k}$; its mean value across all subjects is 0 , with variance $\sigma_{* k}^{2} ; x_{i j}=X_{i j}-\bar{X}$, the deviation of observed log odds for subject $j$ in test $i$ from the overall mean (the $X_{i j}$ values are fixed); $\beta_{k}=$ the effect on the location of the $k$ th coordinate per unit change in $X_{i j}$; and $\varepsilon_{i j k}=$ a within-subject error $\left(0, \sigma_{r k}^{2}\right)$ in dimension $k$, such that

\section{APPENDIX (Continued)}

$$
\sum_{k} \varepsilon_{i j k}^{2}
$$

is distributed as a squared randomly observed normal variable.

\section{Reliability}

Observed coordinate and covariate values in each dimension are processed and presented by standard computer software (SYSTAT) with sum of squares $(S S)$ and mean square $(M S)$ ANCOVA format for $k=1,2, \ldots, 5$ as follows:

$\begin{array}{llll}\text { Source } & d f & S S_{k} & M S_{k} \\ \text { Subjects } & n-1 & S S_{s k} & M S_{s k} \\ \text { Performance } & 1 & S S_{p k} & M S_{p k} \\ \text { Error } & n-1 & S S_{r k} & M S_{r k}\end{array}$

Summing over $k$ (indicated by dot notation) produces the following ANCOVA:

$\begin{array}{lllll}\text { Source } & d f & S S . & M S . & E M S . \\ \text { Subjects } & n-1 & S S_{s .} & M S_{s .} & 2 \sigma_{s .}^{2}+\sigma_{r}^{2} \\ \text { Performance } & 1 & S S_{p .} & M S_{p .} & \sigma_{p .}^{2}+\sigma_{r}^{2} \\ \text { Error } & n-1 & S S_{r .} & M S_{r .} & \sigma_{r .}^{2} .\end{array}$

By definition, performance-corrected reliability is given by

$$
\rho^{2}=\sigma_{s}^{2} /\left(\sigma_{s .}^{2}+\sigma_{r}^{2}\right)
$$

This is the partial regression multidimensional extension of Snedecor and Cochran's (1989) one-dimensional definition.

Since $M S_{s}$ estimates $2 \sigma_{s}^{2}+\sigma_{r}^{2}$, and $M S_{r}$ estimates $\sigma_{r}^{2}, M S_{s .}-$ $M S_{r}$ estimates $2 \sigma_{s .}^{2}$. Similarly, $M S_{s .}+M S_{r}$ estimates $2\left(\sigma_{s .}^{2}+\right.$ $\sigma_{r}^{2}$ ). Consequently, the estimate of $\rho^{2}$ is the ratio of the latter two values:

$$
R^{2}=\left(M S_{s .}-M S_{r}\right) /\left(M S_{s .}+M S_{r}\right)
$$

In this special case of equal degrees of freedom for both subjects and error variance estimates, $R^{2}$ is an mle of reliability.

\section{5\% Confidence Limits}

Letting $\left(2 \sigma_{s}^{2}+\sigma_{r}^{2}\right) / \sigma_{r}^{2}=C, M S_{s} / M S_{r} C$ is distributed as $F$ with $(n-1)$ numerator and denominator degrees of freedom. Consequently, letting $F_{.025}$ and $F_{.975}$ refer to the lower and upper tail critical values of $F$, the lower and upper $95 \%$ confidence limits for $C$ are, respectively,

$$
C_{\mathrm{L}}=M S_{s .} / M S_{r} F_{.975}
$$

and

$$
C_{\mathrm{U}}=M S_{s .} / M S_{r} F_{.025}
$$

The mle of reliability can be re-expressed as

$$
R^{2}=\left(M S_{s} / M S_{r}-1\right) /\left(M S_{s} / M S_{r}+1\right) .
$$

Thus, the lower and upper $95 \%$ confidence limits are given by

$$
R_{\mathrm{L}}^{2}=\left(C_{\mathrm{L}}-1\right) /\left(C_{\mathrm{L}}+1\right)
$$

and

$$
R_{\mathrm{U}}^{2}=\left(C_{\mathrm{U}}-1\right) /\left(C_{\mathrm{U}}+1\right)
$$

These formulas determine the confidence limits reported in the main text.

(Manuscript received September 9, 1998; revision accepted for publication June 29, 1999.) 